Check for updates

Cite this: RSC Adv., 2019, 9, 38658

Received 23rd August 2019

Accepted 21st November 2019

DOI: $10.1039 / \mathrm{c} 9 \mathrm{ra} 06635 \mathrm{~g}$

rsc.li/rsc-advances

\title{
UPLC-QTOF-MS-guided isolation of anti-COPD ginsenosides from wild ginseng $\dagger$
}

\author{
Hailin Zhu, ${ }^{a}$ Junli Liu, ${ }^{a}$ Hongqiang Lin, ${ }^{a}$ Ying Zhang, ${ }^{\text {ac }}$ Na Yang, ${ }^{a}$ Baisong Zhou, \\ Zhongyao Wang, ${ }^{a}$ Alan Chen-Yu Hsu, ${ }^{d}$ Jinping Liu (D)*ab and Pingya Li ${ }^{\star a}$
}

Four previously undescribed ginsenosides, along with five known analogues were isolated from wild ginseng by a UPLC-QTOF-MS-guided fractionation procedure. Their structures were elucidated on the basis of spectroscopic and spectrometric data (1D and 2D NMR, HR-ESI-MS). The isolated compounds could significantly inhibit the cigarette smoke extract (CSE)-induced inflammatory reaction in A549 cells. The HDAC2 pathway might be involved in the protective effect against the CSE-mediated inflammatory response in A549 cells.

\section{Introduction}

Panax ginseng has received much attention not only as a medicinal herb but also as a functional food. ${ }^{1}$ Ginseng has a wide range of biological activities such as anti-inflammatory, anti-oxidant and immunomodulatory functions. It is the traditional belief that wild ginseng is more medicinally efficacious and more valuable than cultivated ginseng. In the past, natural wild ginseng has been used to refer to the original ecological ginseng. But it is hard to find the natural wild ginseng because it is nearly extinct now. Currently, according to the "Identification and Grade Quality Standards of Wild Ginseng" (National Standards of People's Republic of China, GB/T 18765-2015), wild ginseng refers to the ginseng cultivated in mountains and grown for more than 15 years in a natural environment without any human intervention. This kind of wild ginseng is a replacement for natural wild ginseng grown within the original ecological environment. It has been reported that there were differences in immunomodulating effects between wild ginseng and cultivated ginseng. ${ }^{2}$ In our previous study, it was also found that the wild ginseng (20 year-old mountaincultivated ginseng) was much differentiated from cultivated ginseng. And based on UPLC-QTOF/MS, a total of 126 chemical compositions were tentatively identified or characterized from wild ginseng. ${ }^{3}$

${ }^{a}$ School of Pharmaceutical Sciences, Jilin University, Fujin Road 1266, Changchun 130021, Jilin, China. E-mail: liujp@jlu.edu.cn; lipy@jlu.edu.cn; Tel: +86-431-85619803

${ }^{b}$ Research Center of Natural Drug, Jilin University, Changchun 130021, Jilin, China ${ }^{c}$ The First Hospital of Jilin University, Changchun 130021, Jilin, China

${ }^{d}$ Priority Research Centre for Healthy Lungs, Faculty of Health and Medicine, The University of Newcastle, Newcastle, NSW, Australia

$\dagger$ Electronic supplementary information (ESI) available: ${ }^{1} \mathrm{H}$ NMR, ${ }^{13} \mathrm{C}$ NMR, HMQC, HMBC, HR-ESI-MS of compound 1-9. See DOI: 10.1039/c9ra06635g
Chronic Obstructive Pulmonary Disease (COPD), the fourth leading cause of death in the world currently, is usually caused by significant exposure to noxious gases or particles. The airflow limitation and persistent respiratory symptoms are the typical symptoms. Cigarette smokers have a higher prevalence of respiratory symptoms and higher COPD mortality rate, and the cigarette smoking is the leading environmental risk factor for COPD around the world. ${ }^{4}$ Along with the progressive lung inflammation, some pro-inflammatory mediators such as TNF$\alpha, \mathrm{IL}-1 \beta$ and IL-6 participated in the occurrence and development of COPD., ${ }^{5,6}$ Ginsenosides are regarded as the main bioactive components in ginseng. ${ }^{7,8}$ Ginseng extract ${ }^{9,10}$ and monomer saponins such as $\mathrm{Rg}_{1}$ (ref. 11), $\mathrm{Rh}_{2}$ (ref. 12) and $\mathrm{CK}^{13}$ had been reported to significantly inhibit COPD-related inflammation in vivo and in vitro. Reduced HDAC2 activity and expression was found in COPD, resulting in amplification of the inflammatory response. Meanwhile, HDAC2 dysfunction is thought to play an important role in the development of corticosteroid resistance in COPD. ${ }^{14-16}$ Thus, increasing HDAC2 activity may be a promising strategy to overcome corticosteroid resistance in COPD. Existing treatments such as theophylline, nortriptyline, macrolides and selective phosphatidylinositol-3kinase- $\delta$ inhibitors have been reported to increase HDAC2 activity effectively. ${ }^{17}$

As part of our continuing efforts to elucidate the chemical composition of wild ginseng furtherly and search for anti-COPD compounds from wild ginseng, a UPLC-QTOF-MS-guided fractionation procedure was performed to target ginsenosides from the anti-COPD fractions of wild ginseng, leading to the isolation of 4 previously unreported ginsenosides (1-4) and five known analogues (5-9). Herein, the isolation and structural elucidation of the isolated compounds as well as their anti-COPD activity on the CSE-stimulated A549 cells were discussed. The potential mechanism against the CSE stimulation was also preliminarily investigated in the paper. 


\section{Experimental}

\subsection{Plant materials}

The 20 years-old mountain-cultivated ginsengs were collected from cultivation area in Fusong, Jilin Province, the main source of ginseng in China. The ginseng was identified by our group according to Chinese Pharmacopoeia (2015 version). The voucher specimen (no. MCG170931) was deposited at the Research Center of Natural Drug, Jilin University, Changchun, China.

\subsection{Apparatus and chemicals}

The HR-ESI-MS spectra were performed on Waters Xevo G2-XS QTOF mass spectrometer (Waters, Milford, MA, USA). NMR spectra were measured on a Bruker Avance-600 spectrometer (Bruker, Karlsruhe, Germany) in $\mathrm{DMSO}^{-\mathrm{D}_{6}}$ operating at 600 $\mathrm{MHz}$ for ${ }^{1} \mathrm{H}$ NMR, HMBC and HMQC, $150 \mathrm{MHz}$ for ${ }^{13} \mathrm{C} \mathrm{NMR}$, respectively. Tetramethylsilane (TMS) was used as internal standard. Column chromatography was performed with silica gel (200-300 mesh) purchased from Qingdao Ocean Chemical Group Co. Ltd (Qingdao, China). Thin-layer chromatography was conducted on silica gel G plates (Qingdao Marine Chemical Inc.). Semi-preparative HPLC with 1525 binary pump combined Waters 2998 UV detector (Waters Co., Milford, MA, USA.) and YMC C $_{18}$ column $(5 \mu \mathrm{m}, 20 \mathrm{~mm} \times 10 \mathrm{~mm}$; YMC Co., Ltd., Japan) were also used to isolate the compounds. Methanol (MeOH) and acetonitrile (ACN) were HPLC grade (Fisher, USA). Purified water was purchased from YiBao Co, Ltd (Shenzhen, China). Other solvents used were analytical grade (Beijing Chemical Works, Beijing, China).

\subsection{Extraction and isolation}

The fresh wild ginseng $(1.0 \mathrm{~kg})$ were crushed and extracted with 95\% EtOH for three times. The extract was combined and then concentrated under vacuum to obtain the concentrated aqueous solution $(0.5 \mathrm{~L})$, and successively partitioned with petroleum ether, EtOAc and $n$-butanol, to afford petroleum ether (29.8 g), EtOAc (35.2 g) and $n$-butanol (133.5 g) soluble extracts, respectively. The $n$-butanol extract (inhibition of the release of proinflammatory mediators in CSE-induced A549 cells at $200 \mu \mathrm{M}$, Fig. $\mathrm{S} 10 \mathrm{~A} \dagger$ ) was subjected to rapid medium pressure purification chromatography on silica gel (200-300 mesh) eluted with a gradient of chloroform-methanol $(1: 0-0: 1)$ to give 10 fractions (Fr. A-J). Fraction $\mathrm{G}$ showed significant inhibition of the release of pro-inflammatory mediators in CSE-induced A549 cells at $200 \mu \mathrm{M}$ (Fig. S10B $\dagger$ ). Subsequent UPLC-QTOF-MS-guided separation and purification targeted the subfractions of fraction G containing compounds with $\mathrm{m} / z>500$. Fraction $\mathrm{G}$ was further fractionated on silica gel column chromatography eluting with gradient elution of chloroform-methanol (10:1-1:1) to yield six subfractions (G1-G6, Fig. S10C $\dagger$ ). Subfraction G5 (0.21 g) was further separated by semi-preparative RP-HPLC with $\mathrm{ACN} / \mathrm{H}_{2} \mathrm{O}$ as mobile phase to obtain compound $1\left(\mathrm{ACN}-\mathrm{H}_{2} \mathrm{O}: 19: 81,2\right.$ $\mathrm{mL} \min ^{-1}$, $\left.19 \mathrm{~min}, 6 \mathrm{mg}, 0.0006 \%\right)$, compound $2\left(\mathrm{ACN}-\mathrm{H}_{2} \mathrm{O}\right.$ :

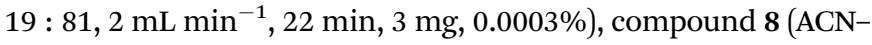
$\mathrm{H}_{2} \mathrm{O}: 21: 79,2 \mathrm{~mL} \min ^{-1}, 5 \mathrm{~min}, 4 \mathrm{mg}, 0.0003 \%$ ), compound 9
(ACN- $\left.\mathrm{H}_{2} \mathrm{O}: 42: 58,2 \mathrm{~mL} \mathrm{~min}{ }^{-1}, 36 \mathrm{~min}, 3 \mathrm{mg}, 0.0003 \%\right)$. G4 (0.14 g) was also further separated by semi-preparative RP-HPLC with $\mathrm{ACN} / \mathrm{H}_{2} \mathrm{O}$ as mobile phase to obtain compound $3\left(\mathrm{ACN}-\mathrm{H}_{2} \mathrm{O}\right.$ : 15 : 85, $2 \mathrm{~mL} \mathrm{~min}^{-1}, 15 \mathrm{~min}, 4 \mathrm{mg}, 0.0004 \%$ ), compound 5 (ACN$\left.\mathrm{H}_{2} \mathrm{O}: 18: 82,2 \mathrm{~mL} \mathrm{~min}^{-1}, 32 \mathrm{~min}, 3 \mathrm{mg}, 0.0003 \%\right)$ and compound

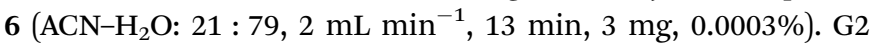
$(0.18 \mathrm{~g})$ was also further separated by semi-preparative RP-HPLC with $\mathrm{ACN} / \mathrm{H}_{2} \mathrm{O}$ as mobile phase to obtain compound 4 (ACN$\mathrm{H}_{2} \mathrm{O}: 21$ : 79, $2 \mathrm{~mL} \mathrm{~min}^{-1}, 10 \mathrm{~min}, 2 \mathrm{mg}, 0.0002 \%$ ) and compound

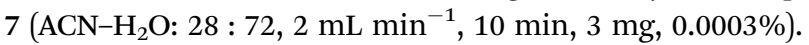

\subsection{Spectroscopic data}

\subsubsection{Ginsenoside-Rm1 (1)}

6-O-[ $\alpha$-L-rhamnopyranosyl- $(1 \rightarrow 2)-\beta-D$-glucopyranosyl]-20-O- $\beta$ - $D^{-}$ glucopyranosyl-dammar-23,25-diene-3 $\beta, 6 \alpha, 12 \beta, 20 S$-tetraol.

White amorphous powder; Libermann-Burchard reaction was positive; Molisch reaction was positive; suggesting the existence of triterpenoid structure; ${ }^{1} \mathrm{H}$ and ${ }^{13} \mathrm{C}$ NMR: see Table 1 ; HR-ESIMS $m / z$ 943.5270 [M - H] $]^{-}$(calc. for $\mathrm{C}_{48} \mathrm{H}_{79} \mathrm{O}_{18}$ is 943.5266); HRMS, ${ }^{1} \mathrm{H}$ and ${ }^{13} \mathrm{C}$ NMR, HMQC, HMBC spectra see in the ESI (Fig. S1-1-S1-5).†

\subsubsection{Ginsenoside-Rm2 (2)}

6-O-[ $\alpha$-L-rhamnopyranosyl- $(1 \rightarrow 2)$ - $\beta$-D-glucopyranosyl $]-20-O-\beta$ - $D$ glucopyranosyl-dammar-22(23),24-diene-3 $\beta, 6 \alpha, 12 \beta, 20 S$-tetraol.

White amorphous powder; Libermann-Burchard reaction was positive; Molisch reaction was positive; suggesting the existence of triterpenoid structure; ${ }^{1} \mathrm{H}$ and ${ }^{13} \mathrm{C}$ NMR: see Table 1 ; HR-ESIMS $m / z$ 943.5272 [M - H] $]^{-}$(calc. for $\mathrm{C}_{48} \mathrm{H}_{79} \mathrm{O}_{18}$ is 943.5266); HRMS, ${ }^{1} \mathrm{H}$ and ${ }^{13} \mathrm{C}$ NMR, HMQC, HMBC spectra see in the ESI (Fig. S2-1-S2-5). $\dagger$

\subsubsection{Ginsenoside-Rm3 (3)}

3-O-[ $\beta$-D-glucopyranosyl- $(1 \rightarrow 2)-\beta$-D-glucopyranosyl]-3 $\beta, 12 \beta,-$ 20S,25-tetrahydroxydammar-23-ene. White amorphous powder; Libermann-Burchard reaction was positive; Molisch reaction was positive; suggesting the existence of triterpenoid structure; ${ }^{1} \mathrm{H}$ and ${ }^{13} \mathrm{C}$ NMR: see Table 2; HR-ESI-MS $m / z$ 799.4897 $[\mathrm{M}-\mathrm{H}]^{-}$ (calc. for $\mathrm{C}_{42} \mathrm{H}_{71} \mathrm{O}_{14}$ is 799.4844); HR-MS, ${ }^{1} \mathrm{H}$ and ${ }^{13} \mathrm{C} \mathrm{NMR}$, HMQC, HMBC spectra see in the ESI (Fig. S3-1-S3-5). $\dagger$

\subsubsection{Ginsenoside-Rm4 (4)}

6-O-[ $\alpha$-L-rhamnopyranosyl- $(1 \rightarrow 2)-\beta$-D-glucopyranosyl]-20-Omalonyl-dammar-24-ene-3 $\beta, 6 \alpha, 12 \beta, 20$-tetraol. White amorphous powder; Libermann-Burchard reaction was positive; Molisch reaction was positive; suggesting the existence of triterpenoid structure; ${ }^{1} \mathrm{H}$ and ${ }^{13} \mathrm{C}$ NMR: see Table 1; HR-ESI-MS $m / z$ 869.4890 [M $-\mathrm{H}]^{-}$(calc. for $\mathrm{C}_{45} \mathrm{H}_{73} \mathrm{O}_{16}$ is 869.4899); HRMS, ${ }^{1} \mathrm{H}$ and ${ }^{13} \mathrm{C}$ NMR, HMQC, HMBC spectra see in the ESI (Fig. S4-1-S4-5).†

\subsubsection{Compound 5-9}

Compound 5-9. White amorphous powder; LibermannBurchard reaction was positive; Molisch reaction was positive; suggesting the existence of triterpenoid structure; ${ }^{1} \mathrm{H}$ and ${ }^{13} \mathrm{C}$ NMR, spectra see in the ESI (Fig. S5-1-S9-2). $\dagger$

\subsection{Bioassay}

2.5.1 Preparation of cigarette smoke extract. In the present study, the cigarettes used were Xiongshi cigarette (China 
Table $1{ }^{1} \mathrm{H}$ and ${ }^{13} \mathrm{C}-\mathrm{NMR}(600 \mathrm{MHz}, 150 \mathrm{MHz}$ in DMSO-D $)$ data for $1,2,4$

\begin{tabular}{|c|c|c|c|c|c|c|}
\hline \multirow[b]{2}{*}{ Position } & \multicolumn{2}{|l|}{1} & \multicolumn{2}{|l|}{2} & \multicolumn{2}{|l|}{4} \\
\hline & $\delta_{\mathrm{C}}$ & $\delta_{\mathrm{H}}$ & $\delta_{\mathrm{C}}$ & $\delta_{\mathrm{H}}$ & $\delta_{\mathrm{C}}$ & $\delta_{\mathrm{H}}$ \\
\hline 1 & 38.73 & $1.44(1 \mathrm{H}, \mathrm{m}), 0.91(1 \mathrm{H}, \mathrm{m})$ & 38.88 & $1.41(1 \mathrm{H}, \mathrm{m}), 0.99(1 \mathrm{H}, \mathrm{m})$ & 38.76 & $1.32(1 \mathrm{H}, \mathrm{m}), 0.92(1 \mathrm{H}, \mathrm{m})$ \\
\hline 2 & 26.80 & $1.51(1 \mathrm{H}, \mathrm{m}), 1.50(1 \mathrm{H}, \mathrm{m})$ & 26.94 & $1.60(1 \mathrm{H}, \mathrm{m}), 1.47(1 \mathrm{H}, \mathrm{m})$ & 26.79 & $1.82(1 \mathrm{H}, \mathrm{m}), 1.75(1 \mathrm{H}, \mathrm{m})$ \\
\hline 3 & 76.90 & $3.54(1 \mathrm{H}, \mathrm{m})$ & 77.37 & $3.51(1 \mathrm{H}, \mathrm{m})$ & 77.21 & $3.65(1 \mathrm{H}, \mathrm{m})$ \\
\hline 4 & 39.98 & - & 39.97 & - & 39.91 & - \\
\hline 5 & 59.86 & $1.40(1 \mathrm{H}, \mathrm{m})$ & 60.01 & $1.45(1 \mathrm{H}, \mathrm{m})$ & 59.84 & $1.07(1 \mathrm{H}, \mathrm{m})$ \\
\hline 6 & 73.84 & $4.66(1 \mathrm{H}, \mathrm{m})$ & 72.73 & $4.63(1 \mathrm{H}, \mathrm{m})$ & 72.63 & $4.53(1 \mathrm{H}, \mathrm{m})$ \\
\hline 7 & 44.75 & $2.17(1 \mathrm{H}, \mathrm{m}), 1.76(1 \mathrm{H}, \mathrm{m})$ & 44.90 & $2.14(1 \mathrm{H}, \mathrm{m}), 1.89(1 \mathrm{H}, \mathrm{m})$ & 44.75 & $2.24(1 \mathrm{H}, \mathrm{m}), 1.90(1 \mathrm{H}, \mathrm{m})$ \\
\hline 8 & 40.16 & - & 40.31 & - & 40.16 & - \\
\hline 9 & 48.64 & $1.42(1 \mathrm{H}, \mathrm{m})$ & 48.79 & $1.39(1 \mathrm{H}, \mathrm{m})$ & 48.72 & $1.17(1 \mathrm{H}, \mathrm{m})$ \\
\hline 10 & 38.59 & - & 38.73 & - & 38.63 & - \\
\hline 11 & 30.27 & $1.92(1 \mathrm{H}, \mathrm{m}), 1.41(1 \mathrm{H}, \mathrm{m})$ & 30.43 & $2.01(1 \mathrm{H}, \mathrm{m}), 1.41(1 \mathrm{H}, \mathrm{m})$ & 30.91 & $2.15(1 \mathrm{H}, \mathrm{m}), 1.07(1 \mathrm{H}, \mathrm{m})$ \\
\hline 12 & 69.08 & $3.86(1 \mathrm{H}, \mathrm{m})$ & 70.32 & $3.87(1 \mathrm{H}, \mathrm{m})$ & 69.81 & $3.90(1 \mathrm{H}, \mathrm{m})$ \\
\hline 13 & 48.06 & $1.69(1 \mathrm{H}, \mathrm{m})$ & 48.61 & $1.73(1 \mathrm{H}, \mathrm{m})$ & 47.03 & $1.96(1 \mathrm{H}, \mathrm{m})$ \\
\hline 14 & 50.80 & - & 50.95 & - & 50.94 & - \\
\hline 15 & 30.09 & $1.40(1 \mathrm{H}, \mathrm{m}), 0.91(1 \mathrm{H}, \mathrm{m})$ & 30.24 & $1.37(1 \mathrm{H}, \mathrm{m}), 0.88(1 \mathrm{H}, \mathrm{m})$ & 30.73 & $1.37(1 \mathrm{H}, \mathrm{m}), 0.86(1 \mathrm{H}, \mathrm{m})$ \\
\hline 16 & 25.90 & $1.48(1 \mathrm{H}, \mathrm{m}), 1.26(1 \mathrm{H}, \mathrm{m})$ & 26.94 & $1.45(1 \mathrm{H}, \mathrm{m}), 1.26(1 \mathrm{H}, \mathrm{m})$ & 26.03 & $1.45(1 \mathrm{H}, \mathrm{m}), 1.07(1 \mathrm{H}, \mathrm{m})$ \\
\hline 17 & 51.05 & $2.50(1 \mathrm{H}, \mathrm{m})$ & 51.20 & $2.47(1 \mathrm{H}, \mathrm{m})$ & 53.49 & $2.30(1 \mathrm{H}, \mathrm{m})$ \\
\hline 18 & 16.74 & $0.82(3 \mathrm{H}, \mathrm{s})$ & 16.80 & $0.91(3 \mathrm{H}, \mathrm{s})$ & 16.97 & $0.83(3 \mathrm{H}, \mathrm{s})$ \\
\hline 19 & 16.84 & $0.81(3 \mathrm{H}, \mathrm{s})$ & 16.89 & $0.78(3 \mathrm{H}, \mathrm{s})$ & 17.56 & $0.83(3 \mathrm{H}, \mathrm{s})$ \\
\hline 20 & 82.02 & - & 82.17 & - & 75.24 & - \\
\hline 21 & 22.37 & $1.22(3 \mathrm{H}, \mathrm{s})$ & 22.10 & $1.19(3 \mathrm{H}, \mathrm{s})$ & 26.38 & $0.97(3 \mathrm{H}, \mathrm{s})$ \\
\hline 22 & 40.01 & $2.13(1 \mathrm{H}, \mathrm{m}), 1.88(1 \mathrm{H}, \mathrm{m})$ & 141.31 & $6.05(1 \mathrm{H}, \mathrm{d}, J=11.5 \mathrm{~Hz})$ & 34.911 & $2.01(1 \mathrm{H}, \mathrm{m}), 1.32(1 \mathrm{H}, \mathrm{m})$ \\
\hline 23 & 127.283 & $5.53(1 \mathrm{H}, \mathrm{dt}, J=12.5,7.5 \mathrm{~Hz})$ & 127.43 & $6.25(1 \mathrm{H}, \mathrm{m})$ & 21.973 & $2.60(1 \mathrm{H}, \mathrm{m}), 2.25(1 \mathrm{H}, \mathrm{m})$ \\
\hline 24 & 134.62 & $6.39(1 \mathrm{H}, \mathrm{d}, J=11.0 \mathrm{~Hz})$ & 121.77 & $6.02(1 \mathrm{H}, \mathrm{d}, J=11.5 \mathrm{~Hz})$ & 125.517 & $5.48(1 \mathrm{H}, \mathrm{m})$ \\
\hline 25 & 141.72 & - & 134.77 & - & 130.16 & - \\
\hline 26 & 114.63 & $4.98(1 \mathrm{H}, \mathrm{m}), 4.89(1 \mathrm{H}, \mathrm{m})$ & 31.12 & $2.25(3 \mathrm{H}, \mathrm{s})$ & 25.56 & $1.56(3 \mathrm{H}, \mathrm{s})$ \\
\hline 27 & 17.79 & $1.83(3 \mathrm{H}, \mathrm{s})$ & 22.52 & $1.91(3 \mathrm{H}, \mathrm{s})$ & 16.57 & $1.22(3 \mathrm{H}, \mathrm{s})$ \\
\hline 28 & 30.97 & $1.62(3 \mathrm{H}, \mathrm{s})$ & 31.12 & $1.92(3 \mathrm{H}, \mathrm{s})$ & 31.00 & $1.63(3 \mathrm{H}, \mathrm{s})$ \\
\hline 29 & 17.79 & $0.94(3 \mathrm{H}, \mathrm{s})$ & 16.99 & $0.99(3 \mathrm{H}, \mathrm{s})$ & 16.74 & $0.96(3 \mathrm{H}, \mathrm{s})$ \\
\hline 30 & 16.65 & $0.81(3 \mathrm{H}, \mathrm{s})$ & 16.80 & $0.78(3 \mathrm{H}, \mathrm{s})$ & 16.46 & $0.82(3 \mathrm{H}, \mathrm{s})$ \\
\hline 6-Glc-1' & 99.91 & $5.11(1 \mathrm{H}, \mathrm{d}, J=7.0 \mathrm{~Hz})$ & 100.06 & $5.09(1 \mathrm{H}, \mathrm{d}, J=7.5 \mathrm{~Hz})$ & 99.94 & $5.35(1 \mathrm{H}, \mathrm{m})$ \\
\hline 6-Glc-2 $2^{\prime}$ & 77.22 & $4.48(1 \mathrm{H}, \mathrm{m})$ & 77.37 & $4.40(1 \mathrm{H}, \mathrm{m})$ & 77.53 & $4.19(1 \mathrm{H}, \mathrm{m})$ \\
\hline 6-Glc-3' & 77.51 & $4.34(1 \mathrm{H}, \mathrm{m})$ & 78.43 & $4.32(1 \mathrm{H}, \mathrm{m})$ & 77.21 & $4.32(1 \mathrm{H}, \mathrm{m})$ \\
\hline 6-Glc-4' & 70.82 & $4.14(1 \mathrm{H}, \mathrm{m})$ & 70.96 & $4.12(1 \mathrm{H}, \mathrm{m})$ & 70.85 & $4.12(1 \mathrm{H}, \mathrm{m})$ \\
\hline 6-Glc-5' & 76.78 & $3.90(1 \mathrm{H}, \mathrm{brs})$ & 77.37 & $3.66(1 \mathrm{H}, \mathrm{brs})$ & 77.21 & $4.06(1 \mathrm{H}, \mathrm{brs})$ \\
\hline 6-Glc-6 & 61.47 & $4.62(1 \mathrm{H}, \mathrm{m}), 4.43(1 \mathrm{H}, \mathrm{m})$ & 61.62 & $4.45(1 \mathrm{H}, \mathrm{m}), 4.59(1 \mathrm{H}, \mathrm{m})$ & 61.49 & $4.37(1 \mathrm{H}, \mathrm{m}), 4.24(1 \mathrm{H}, \mathrm{m})$ \\
\hline $2^{\prime}$-Rham-1" & 99.91 & $6.32(1 \mathrm{H}, \mathrm{m})$ & 100.06 & $6.42(1 \mathrm{H}, \mathrm{m})$ & 99.87 & $6.59(1 \mathrm{H}, \mathrm{m})$ \\
\hline $2^{\prime}$-Rham-2" & 73.65 & $5.06(1 \mathrm{H}, \mathrm{m})$ & 70.63 & $4.85(1 \mathrm{H}, \mathrm{m})$ & 70.50 & $4.60(1 \mathrm{H}, \mathrm{m})$ \\
\hline $2^{\prime}$-Rham- $3^{\prime \prime}$ & 70.48 & $4.87(1 \mathrm{H}, \mathrm{m})$ & 70.32 & $4.84(1 \mathrm{H}, \mathrm{m})$ & 70.20 & $4.47(1 \mathrm{H}, \mathrm{m})$ \\
\hline $2^{\prime}$-Rham- $4^{\prime \prime}$ & 72.05 & $4.35(1 \mathrm{H}, \mathrm{m})$ & 72.73 & $4.31(1 \mathrm{H}, \mathrm{m})$ & 72.06 & $4.12(1 \mathrm{H}, \mathrm{m})$ \\
\hline $2^{\prime}-$ Rham-5 ${ }^{\prime \prime}$ & 67.84 & $4.88(1 \mathrm{H}, \mathrm{m})$ & 67.99 & $4.84(1 \mathrm{H}, \mathrm{m})$ & 67.88 & $4.94(1 \mathrm{H}, \mathrm{m})$ \\
\hline $2^{\prime}$-Rham-6 ${ }^{\prime \prime}$ & 16.74 & $1.58(3 \mathrm{H}, \mathrm{d}, J=6.5 \mathrm{~Hz})$ & 17.94 & $1.60(3 \mathrm{H}, \mathrm{d}, J=6.5 \mathrm{~Hz})$ & 17.80 & $1.34(3 \mathrm{H}, \mathrm{d}, J=6.5 \mathrm{~Hz})$ \\
\hline $20-$ Glc-1" ${ }^{\prime \prime \prime}$ & 96.62 & $4.91(1 \mathrm{H}, \mathrm{d}, J=7.5 \mathrm{~Hz})$ & 96.77 & $5.03(1 \mathrm{H}, \mathrm{d}, J=7.5 \mathrm{~Hz})$ & - & - \\
\hline $20-$ Glc- $2^{\prime \prime \prime}$ & 73.84 & $3.66(1 \mathrm{H}, \mathrm{m})$ & 74.00 & $3.59(1 \mathrm{H}, \mathrm{m})$ & - & - \\
\hline $20-$ Glc- $3^{\prime \prime \prime}$ & 77.36 & $4.18(1 \mathrm{H}, \mathrm{m})$ & 77.66 & $4.15(1 \mathrm{H}, \mathrm{m})$ & - & - \\
\hline $20-$ Glc- $4^{\prime \prime \prime}$ & 69.28 & $3.84(1 \mathrm{H}, \mathrm{m})$ & 69.72 & $3.87(1 \mathrm{H}, \mathrm{m})$ & - & - \\
\hline $20-$ Glc-5 ${ }^{\prime \prime \prime}$ & 76.68 & $3.62(1 \mathrm{H}, \mathrm{m})$ & 77.37 & $3.51(1 \mathrm{H}, \mathrm{m})$ & - & - \\
\hline $20-$ Glc-6 $6^{\prime \prime \prime}$ & 61.23 & $4.56(1 \mathrm{H}, \mathrm{m}), 4.26(1 \mathrm{H}, \mathrm{m})$ & 61.38 & $4.53(1 \mathrm{H}, \mathrm{m}), 4.23(1 \mathrm{H}, \mathrm{m})$ & - & - \\
\hline$-\mathrm{O}-\mathrm{CO}-$ & - & - & - & - & 170.90 & - \\
\hline$-\mathrm{CH}_{2}$ & - & - & - & - & 38.76 & $3.77(2 \mathrm{H}, \mathrm{m})$ \\
\hline$-\mathrm{CO}-\mathrm{OH}$ & - & - & - & - & 171.20 & - \\
\hline
\end{tabular}

Tobacco Zhejiang Industrial Co., Ltd, Hangzhou, China) containing $11 \mathrm{mg}$ of tar, $0.7 \mathrm{mg}$ of nicotine and $13 \mathrm{mg}$ of carbon monoxide per cigarette. Cigarette smoke extract (CSE) was prepared essentially as reported previously. ${ }^{18-20}$ Smoke from one cigarette was bubbled into $20 \mathrm{~mL}$ of culture medium $(300 \mathrm{~s}$ per cigarette). The CSE solution was filtered through a $0.22 \mu \mathrm{m}$ sterile filter after being incubated at $37^{\circ} \mathrm{C}$ for $30 \mathrm{~min}$. The CSE solution was prepared freshly and was used within half an hour. This CSE solution was regarded as the highest concentration $(100 \%)$.

2.5.2 Cell viability assay. Human lung carcinoma A549 cells were obtained from the Department of Pathogen Biology, Basic Medical College, Jilin University. Each ginsenoside was dissolved in DMSO to obtain the stock solution stored in $4{ }^{\circ} \mathrm{C}$. The 
Table $2{ }^{1} \mathrm{H}$ and ${ }^{13} \mathrm{C}-\mathrm{NMR}(600 \mathrm{MHz}, 150 \mathrm{MHz}$ in DMSO-D $)$ data for $3^{a}$

\begin{tabular}{|c|c|c|c|c|c|}
\hline \multirow[b]{2}{*}{ Position } & \multicolumn{2}{|l|}{3} & \multirow[b]{2}{*}{ Position } & \multicolumn{2}{|l|}{3} \\
\hline & $\delta_{\mathrm{C}}$ & $\delta_{\mathrm{H}}$ & & $\delta_{\mathrm{C}}$ & $\delta_{\mathrm{H}}$ \\
\hline 2 & 25.79 & $2.25(1 \mathrm{H}, \mathrm{m}), 1.55(1 \mathrm{H}, \mathrm{m})$ & 23 & 126.20 & $6.31(1 \mathrm{H}, \mathrm{m})$ \\
\hline 3 & 88.22 & $3.15(1 \mathrm{H}, \mathrm{m})$ & 24 & 39.50 & $2.76(1 \mathrm{H}, \mathrm{m}), 2.44(1 \mathrm{H}, \mathrm{m})$ \\
\hline 4 & 39.60 & - & 25 & 80.34 & - \\
\hline 7 & 34.42 & $1.36(1 \mathrm{H}, \mathrm{m}), 1.33(1 \mathrm{H}, \mathrm{m})$ & 28 & 27.48 & $0.98(3 \mathrm{H}, \mathrm{s})$ \\
\hline 8 & 40.02 & - & 29 & 15.99 & $0.90(3 \mathrm{H}, \mathrm{s})$ \\
\hline 9 & 49.44 & $1.31(1 \mathrm{H}, \mathrm{m})$ & 30 & 16.48 & $0.81(3 \mathrm{H}, \mathrm{s})$ \\
\hline 10 & 36.29 & - & 3-Glc-1' & 103.66 & $4.96(1 \mathrm{H}, \mathrm{d}, J=7.5 \mathrm{~Hz})$ \\
\hline 11 & 30.98 & $1.92(1 \mathrm{H}, \mathrm{m}), 1.63(1 \mathrm{H}, \mathrm{m})$ & 3-Glc-2' & 81.35 & $4.02(1 \mathrm{H}, \mathrm{m})$ \\
\hline 12 & 70.02 & $3.30(1 \mathrm{H}, \mathrm{m})$ & 3-Glc-3' & 76.16 & $3.99(1 \mathrm{H}, \mathrm{m})$ \\
\hline 17 & 52.97 & $2.30(1 \mathrm{H}, \mathrm{m})$ & $2^{\prime}-\mathrm{Glc}-2^{\prime \prime}$ & 75.30 & $3.42(1 \mathrm{H}, \mathrm{m})$ \\
\hline 18 & 15.37 & $0.82(3 \mathrm{H}, \mathrm{s})$ & $2^{\prime}-\mathrm{Glc}-3^{\prime \prime}$ & 76.88 & $4.14(1 \mathrm{H}, \mathrm{m})$ \\
\hline 19 & 15.93 & $0.74(3 \mathrm{H}, \mathrm{s})$ & $2^{\prime}-\mathrm{Glc}-4^{\prime \prime}$ & 70.02 & $4.25(1 \mathrm{H}, \mathrm{m})$ \\
\hline 20 & 72.29 & - & $2^{\prime}-$ Glc- $5^{\prime \prime}$ & 76.55 & $3.42(1 \mathrm{H}, \mathrm{m})$ \\
\hline 21 & 27.48 & $1.02(3 \mathrm{H}, \mathrm{s})$ & $2^{\prime}-$ Glc- $6^{\prime \prime}$ & 60.89 & $4.42(1 \mathrm{H}, \mathrm{m}), 4.26(1 \mathrm{H}, \mathrm{m})$ \\
\hline
\end{tabular}

final needed concentration of each compound was acquired by being diluted with Dulbecco's Modified Eagle Medium (DMEM). The growth-inhibition effect of CSE and the effect of new ginsenosides on cell viability of A549 cells were evaluated by MTT assay. ${ }^{21}$

A549 cells were cultured in 96-well plates at a density of $5 \times$ $10^{5}$ cells per well treated with the presence of CSE $(5 \%, 10 \%$, $20 \%, 30 \%$ and $40 \%$ ) for $18 \mathrm{~h}$, or treated with ginsenosides at the concentrations of $0.0,10.0,20.0,40.0,80.0 \mu \mathrm{M}$ for $24 \mathrm{~h}$.

2.5.3 Drug treatment. For all group, A549 cells were cultured for $18 \mathrm{~h}$ in 96 -well plates at a density of $5 \times 10^{5}$ cells per $\mathrm{mL}$. In control group, A549 cells were cultured normally without the CSE stimulation. In CSE group, the cells were stimulated with a certain amount of CSE without any drug intervened. In ginsenoside groups, the cells were treated simultaneously with CSE and 10, 20, 40, and $80 \mu \mathrm{M}$ of each ginsenoside.

2.5.4 Enzyme-linked immunosorbent assay. IL-1 $\beta$, IL-6 and TNF- $\alpha$ contents in the cell culture supernatant were determined with ELISA kits (Nanjing Jiancheng Bioengineering Institute) after $18 \mathrm{~h}$ of incubation. All procedures were performed following the manufacturer's instructions.

2.5.5 Western blotting. Western blotting was performed as previous described $^{22}$ to discuss the effects of ginsenosides on HDAC2 expression in CSE-stimulated A549 cells. Both antiHDAC2 (\#ab32117) and anti- $\beta$-actin (\#ab137550) were purchased from Abcam Company (Cambridge, UK). The HDAC2 band intensities were compared with reference to $\beta$-actin control.

2.5.6 Statistical analysis. Graphpad Prism 6.0 software (CA, USA) was used for all statistical analysis. The results are expressed as $\bar{x} \pm$ SD. Statistical significance was calculated with two tailed test or a one-way analysis of variance (ANOVA), and $p$ value $<0.05$ was considered as statistically significant.

2.5.7 Molecular docking assay. The GLIDE 6.7 software and Maestro Elements 2.2 software (Schrödinger, New York, NY, USA) were applied to perform molecular docking. Firstly, the 3D structures of ginsenosides were acquired respectively; secondly, the ginsenosides' bond angles and orders were then assigned using LigPrep module; thirdly, the 3D X-ray crystal structure of HDAC2 (PDB ID: 5IWG) was retrieved from Protein Data Bank (PDB) and was optimized according to the processes previous reported. ${ }^{23}$ Fourthly, the docking were performed with the following main parameters: the energy of conjugate of ligand and receptor was minimized to a root-mean-square deviation of $0.30 \AA$ in optimized potentials for liquid simulations 3 force field, and the grid box at active site was set at the size of $20 \AA$. Finally, the highest XPG score pose was chosen as the dominant binding form and the visual analysis was performed by PyMol (Schrödinger) software.

\section{Results and discussion}

\subsection{Structure elucidation}

The 95\% EtOH extract of wild ginseng was suspended in aqueous solution and partitioned with petroleum ether, EtOAc and $n$-butanol. UPLC-QTOF-MS-guided isolation of bioactive $n$ butanol soluble extracts (inhibition of the release of proinflammatory mediators in CSE-induced A549 cells at $200 \mu \mathrm{M}$ ) led to identify nine ginsenosides (Fig. S10A $\dagger$ ). Five known compounds were identified as ginsenoside $\mathrm{Rb} 2(5),{ }^{24} \mathrm{Rd}(6),{ }^{24}$ Rg3 (7), ${ }^{25} \operatorname{Rg} 1(8)^{25}$ and Rh2 $(9)^{26}$ by comparing spectroscopic data with those of reported values. 


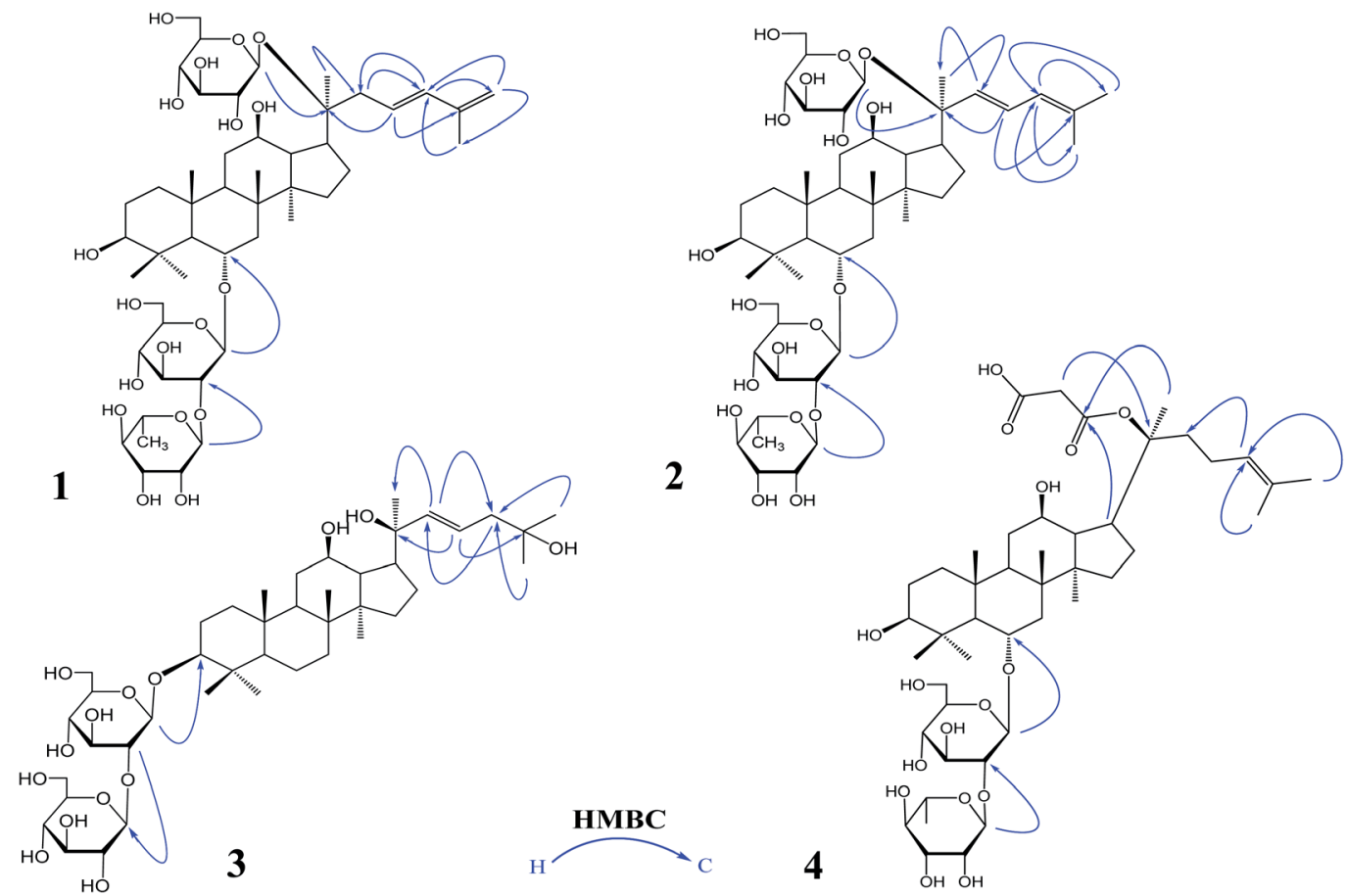

Fig. 1 The important HMBC correlations of 1-4: ginsenoside Rm1 (1); ginsenoside Rm2 (2); ginsenoside Rm3 (3); ginsenoside Rm4 (4) HMBC: heteronuclear multiple bond correlation.

Compound 1 was a white amorphous powder ( $\mathrm{MeOH})$. The molecular formula was established as $\mathrm{C}_{48} \mathrm{H}_{80} \mathrm{O}_{18}$ by combining NMR spectra and HR-ESI-MS at $m / z 943.5270[\mathrm{M}-\mathrm{H}]^{-}$(calc. for 943.5266). The ${ }^{1} \mathrm{H}$ NMR spectrum of 1 displayed eight methyl signals at $\delta 0.81(3 \mathrm{H}, \mathrm{s}), 0.81(3 \mathrm{H}, \mathrm{s}), 0.82(3 \mathrm{H}, \mathrm{s}), 0.94(3 \mathrm{H}, \mathrm{s})$, $1.22(3 \mathrm{H}, \mathrm{s}), 1.58(3 \mathrm{H}, \mathrm{d}, J=6.5 \mathrm{~Hz}), 1.62(3 \mathrm{H}, \mathrm{s})$ and $1.83(3 \mathrm{H}, \mathrm{s})$. The ${ }^{13} \mathrm{C}$ NMR spectrum of 1 showed 48 signals, including ten methylene [two of them bearing an oxygen atom respectively $(\delta$ $61.23,61.47)$, and one of them was olefinic signal at $\delta 114.63]$, twenty-four methine [eighteen of them bearing an oxygen atom respectively $(\delta 67.84,69.08,69.28,70.48,70.82,72.05,73.65$, $73.84,73.84,76.68,76.78,76.90,77.22,77.36,77.51,96.62,99.91$ and 99.91), and two of them were olefinic signals at $\delta 127.28$ and 134.62], six quaternary carbon $[\delta 38.59,39.98,40.16,50.80$, 82.02 and an olefinic signal at $\delta 141.72]$, eight methyl carbons $(\delta$ $16.65,16.74,16.74,16.84,17.79,17.79,22.37$ and 30.97). There is a resemblance with the chemical shifts of 1 along with ginsenoside $\mathrm{Re}^{27}$ except the signals of the side chain. The main differences were the replacement of two olefinic carbons by four olefinic carbons ( $\delta 141.72,134.62,127.28$ and 114.63), and only two methyl signals ( $\delta 22.37$ and 17.79 ) in 1. Furthermore, comparison of the NMR spectroscopic data of $\mathbf{1}$ with those of cylindrictone $A,{ }^{28}$ whose structure was $12 \beta, 20 \beta$-dihydroxy-23,25diene-3-oxo-dammarane, a 23,25-diene group was deduced to exist in compound $\mathbf{1}$. The location of this group was confirmed by the heteronuclear multiple bond coherence (HMBC) spectrum of 1 . The correlations between $\mathrm{H}-22$ with $\mathrm{C}-21$ and $\mathrm{C}-24, \mathrm{H}-$ 23 with C-20 and C-25, H-24 with C-22, C-26 and C-27, H-26 with $\mathrm{C}-24$ and $\mathrm{C}-27, \mathrm{H}-27$ with $\mathrm{C}-24$ and $\mathrm{C}-26$, were observed in the HMBC spectrum of $\mathbf{1}$. The structure of 1 were deduced as $6-O-[\alpha-$ L-rhamnopyranosyl-( $1 \rightarrow 2)-\beta$-D-glucopyranosyl]-20-O- $\beta$-D- glucopyranosyl-dammar-23,25-diene-3 $3,6 \alpha, 12 \beta, 20 S$-tetraol (ginsenoside Rm1, Fig. 1). Compound 1 was a minor glucoside in wild ginseng. The carbon and proton signals of 1 were fully assigned on the basis of HMQC and HMBC spectra (Table 1 and Fig. 2).
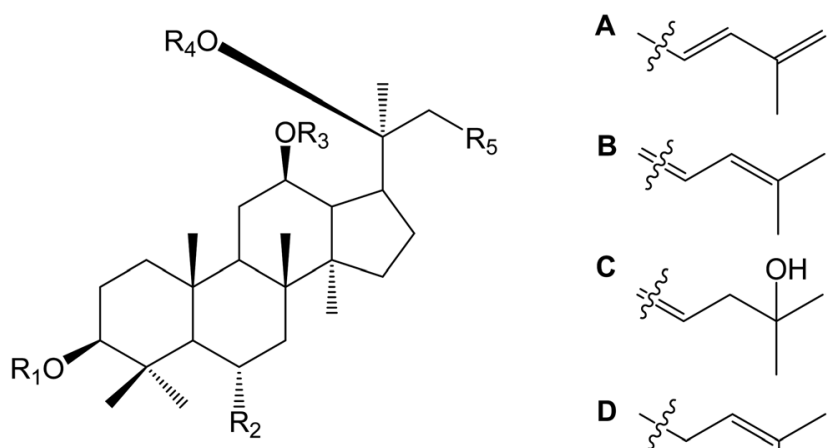

C<smiles>CCCC(C)(C)O</smiles><smiles></smiles>

$1 R_{1}=R_{3}=H, R_{2}=$ Glc $^{2}{ }^{1}$ Rha, $R_{4}=$ Glc, $R_{5}=A$

$2 \mathrm{R}_{1}=\mathrm{R}_{3}=\mathrm{H}, \mathrm{R}_{2}=\mathrm{Glc}^{2}{ }^{1} \mathrm{Rha}, \mathrm{R}_{4}=\mathrm{Glc}, \mathrm{R}_{5}=\mathrm{B}$

$3 \mathbf{R}_{1}=\mathrm{Glc}^{2}{ }_{-}{ }^{\mathrm{Gle}}, \mathrm{R}_{2}=\mathrm{R}_{3}=\mathrm{R}_{4}=\mathrm{H}, \mathrm{R}_{5}=\mathrm{B}$

$4 \mathbf{R}_{1}=\mathbf{R}_{3}=\mathrm{H}, \mathbf{R}_{\mathbf{2}}=\mathrm{Glc}^{2}{ }_{-}{ }^{1} \mathbf{R h a}, \mathbf{R}_{5}=\mathrm{D}$

$5 \mathrm{R}_{1}=\mathrm{Glc}^{2}{ }_{-1}{ }_{\mathrm{Glc}}, \mathrm{R}_{2}=\mathrm{R}_{3}=\mathrm{H}, \mathrm{R}_{4}=\mathrm{Glc}^{6}{ }_{-}{ }^{1} \mathrm{Glc}, \mathrm{R}_{5}=\mathrm{D}$

$6 \mathrm{R}_{1}=\mathrm{Glc}^{2}{ }_{-}{ }^{\mathrm{Glc}}, \mathrm{R}_{2}=\mathrm{R}_{3}=\mathrm{H}, \mathrm{R}_{4}=\mathrm{Glc}, \mathrm{R}_{5}=\mathrm{D}$

$7 \mathrm{R}_{\mathbf{1}}=\mathrm{Glc}^{2}{ }_{-}{ }^{1} \mathrm{Glc}, \mathrm{R}_{2}=\mathrm{R}_{3}=\mathrm{R}_{4}=\mathrm{H}, \mathrm{R}_{5}=\mathrm{D}$

$8 \mathbf{R}_{\mathbf{1}}=\mathbf{R}_{\mathbf{3}}=\mathrm{H}, \mathbf{R}_{\mathbf{2}}=$ Glc, $\mathbf{R}_{\mathbf{4}}=$ Glc, $\mathbf{R}_{\mathbf{5}}=\mathrm{D}$

$9 \mathbf{R}_{\mathbf{1}}=\mathrm{Glc}, \mathrm{R}_{\mathbf{2}}=\mathrm{R}_{\mathbf{3}}=\mathrm{R}_{\mathbf{4}}=\mathrm{H}, \mathrm{R}_{\mathbf{5}}=\mathrm{D}$

Fig. 2 Chemical structures of 1-9: ginsenoside Rm1 (1); ginsenoside $\mathrm{Rm} 2$ (2); ginsenoside Rm3 (3); ginsenoside Rm4 (4); ginsenoside Rb2 (5); ginsenoside Rd (6); ginsenoside Rg3 (7); ginsenoside Rg1 (8); ginsenoside Rh2 (9). 
$\mathbf{A}$
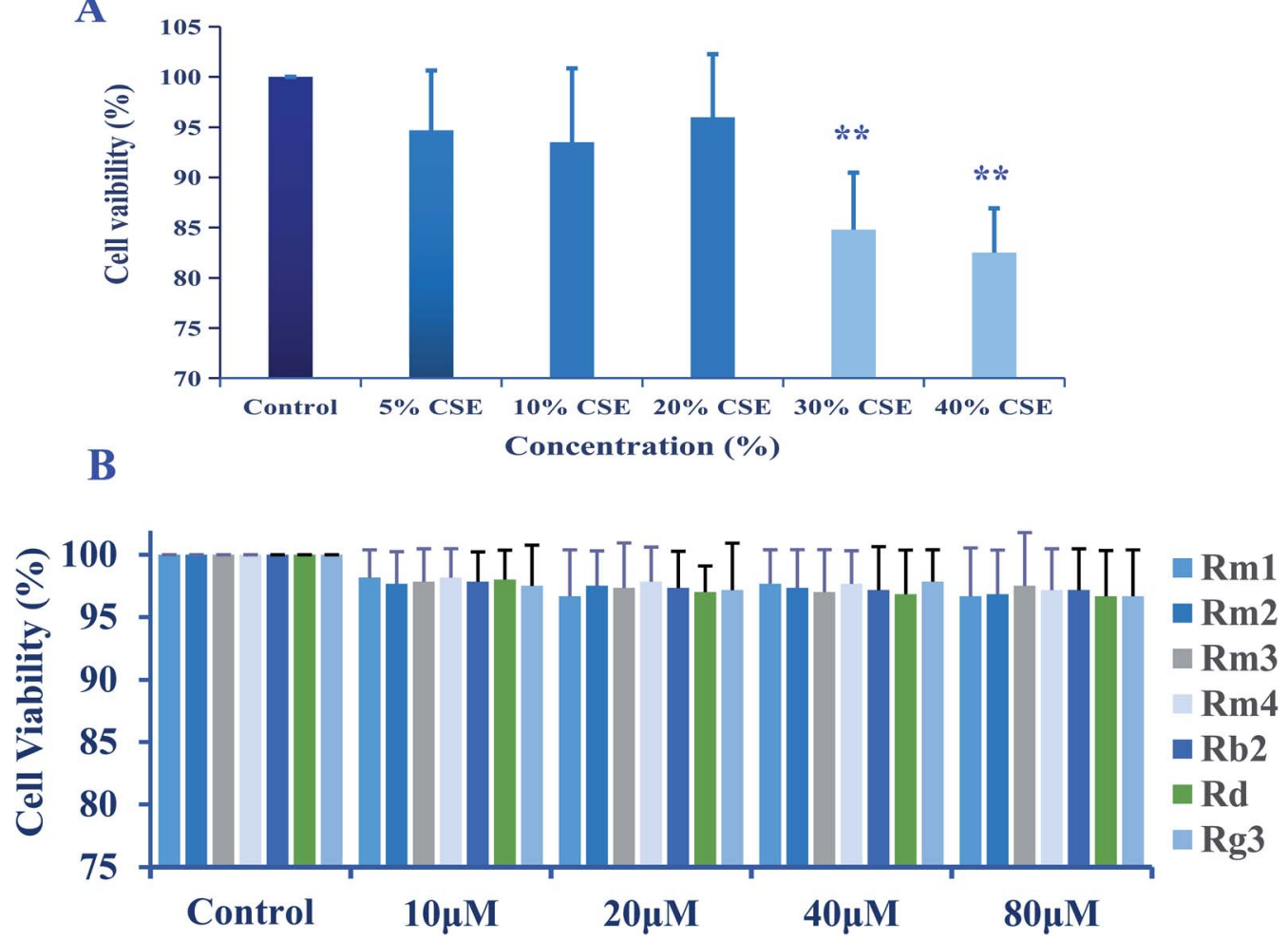

Fig. 3 (A) Cytotoxicity of cigarette smoke extract (CSE) on A549 cells; (B) effect of compound 1-7 on the cell viability of A549 cells. The results represented as mean \pm S.D. $(n=6) . * * 00.01$, compared with control group.

Compound 2 was a white amorphous powder ( $\mathrm{MeOH})$. The molecular formula was established as $\mathrm{C}_{48} \mathrm{H}_{80} \mathrm{O}_{18}$ by combining the NMR spectra and HR-ESI-MS at $m / z 943.5272[\mathrm{M}-\mathrm{H}]^{-}$(calc. for 943.5266 ). The ${ }^{1} \mathrm{H}$ NMR spectrum of 2 displayed nine methyl signals at $\delta 0.78(3 \mathrm{H}, \mathrm{s}), 0.78(3 \mathrm{H}, \mathrm{s}), 0.91(3 \mathrm{H}, \mathrm{s}), 0.99(3 \mathrm{H}, \mathrm{s})$, $1.19(3 \mathrm{H}, \mathrm{s}), 1.60(3 \mathrm{H}, \mathrm{d}, J=6.5 \mathrm{~Hz}), 1.91(3 \mathrm{H}, \mathrm{s}), 1.92(3 \mathrm{H}, \mathrm{s})$ and $2.25(3 \mathrm{H}, \mathrm{s})$. The ${ }^{13} \mathrm{C}$ NMR spectrum of 2 revealed 48 signals, including eight methylene [two of them bearing an oxygen atom respectively $(\delta 61.38,61.62)]$, twenty-five methine [eighteen of them bearing an oxygen atom respectively $(\delta 67.99,69.72,70.32$, $70.32,70.63,70.96,72.73,72.73,74.00,77.37,77.37,77.37$, $77.37,77.66,78.43,96.77,100.06$ and 100.06), and three of them were olefinic signals at $\delta 121.77,127.43$ and 141.31], six quaternary carbon $[\delta 39.97,40.31,38.73,50.95,82.17$ and an olefinic signal at $\delta 134.77]$, nine methyl carbons $(\delta 16.80,16.80$, $16.89,16.99,17.94,22.10,22.52,31.12$ and 31.12$)$. There is a resemblance with the chemical shifts of 2 along with ginsenoside $\mathrm{Re}^{27}$ or compound $\mathbf{1}$ except the signals of the side chain. The main differences were the four olefinic carbons $(\delta 121.77$, $127.43,134.77$ and 141.31), and three methyl signals ( $\delta 22.10$, 22.52 and 31.12) in 2. Furthermore, comparison of the NMR spectroscopic data of 2 with those of notoginsenoside ST14, ${ }^{29}$ whose structure was $(3 \beta, 6 \alpha, 12 \beta, 20 S, 22 E)-3,6,12,20$-tetrahydroxydammar-22(23),24-diene-6-O- $\beta$-D-glucopyranoside, a $22(23), 24-$ diene group was deduced to exist in compound 2 . The location of this group was confirmed by HMBC of 2. In HMBC spectrum, the correlations between $\mathrm{H}-22$ with $\mathrm{C}-21$ and $\mathrm{C}-24, \mathrm{H}-23$ with C20 and C-25, H-24 with C-22, C-26 and C-27, H-26 with C-24 and
C-27, H-27 with C-24 and C-26, were observed. Thus, the structure of compound 2 was identified as $6-O-[\alpha-\mathrm{L}-$-rhamnopyranosyl-( $(1 \rightarrow 2)-\beta$-D-glucopyranosyl]-20-O- $\beta$-D-glucopyranosyldammar-22(23), 24-diene-3 $\beta, 6 \alpha, 12 \beta, 20 S$-tetraol (ginsenoside Rm2, Fig. 1). Compound 2 was also a minor glucoside in wild ginseng. The complete proton and carbon signal assignments were shown in Table 1.

Compound 3, a white amorphous powder $(\mathrm{MeOH})$, possessed a molecular formula of $\mathrm{C}_{42} \mathrm{H}_{72} \mathrm{O}_{14}$, as elucidated by combining the NMR spectra and HR-ESI-MS at $m / z 799.4897$ [M $-\mathrm{H}]^{-}$(calc. for 799.4844). The ${ }^{1} \mathrm{H}$ NMR spectrum of 3 displayed eight methyl signals at $\delta 0.74(3 \mathrm{H}, \mathrm{s}), 0.81(3 \mathrm{H}, \mathrm{s}), 0.82(3 \mathrm{H}, \mathrm{s})$, $0.90(3 \mathrm{H}, \mathrm{s}), 0.98(3 \mathrm{H}, \mathrm{s}), 1.02(3 \mathrm{H}, \mathrm{s}), 1.56(3 \mathrm{H}, \mathrm{s})$ and $1.64(3 \mathrm{H}$, $\mathrm{s})$. The ${ }^{13} \mathrm{C}$ NMR spectrum of 3 revealed 42 signals, including ten methylene [two of them bearing an oxygen atom respectively $(\delta 60.89,61.06)]$, twenty methine [twelve of them bearing an oxygen atom respectively $(\delta 69.88,70.02,70.02,75.30,76.55$, $76.16,76.43,76.88,81.35,88.22,103.6$ and 103.87), and two of them were olefinic signals at $\delta 126.20$ and 136.16], six quaternary carbon $[\delta 36.29,39.60,40.02,50.98,72.29$ and 80.34$]$, eight methyl carbons $(\delta 15.37,15.93,15.99,16.48,27.48,27.48,29.94$ and 29.96). The NMR data of 3 were similar to those of quinquenoside $\mathrm{A},{ }^{29,30}$ whose structure was 3-O-[ $\beta$-D-glucopyranosyl$(1 \rightarrow 2)$ - $\beta$-D-glucopyranosyl]-20-O-[ $\beta$-D-glu-copyranosyl- $(1 \rightarrow 6)-\beta$ D-glucopyranosyl]-3 $3,12 \beta, 20 S, 25$-tetrahydroxydammar-23-ene, except the absence of $\beta$-D-glucopyranosyl-( $1 \rightarrow 6)$ - $\beta$-D-glucopyranosyl group signals in 3 . And the chemical shift of C-20 was changed to $\delta 72.29$, indicated that $\mathrm{C}-20$ was linked to a free 

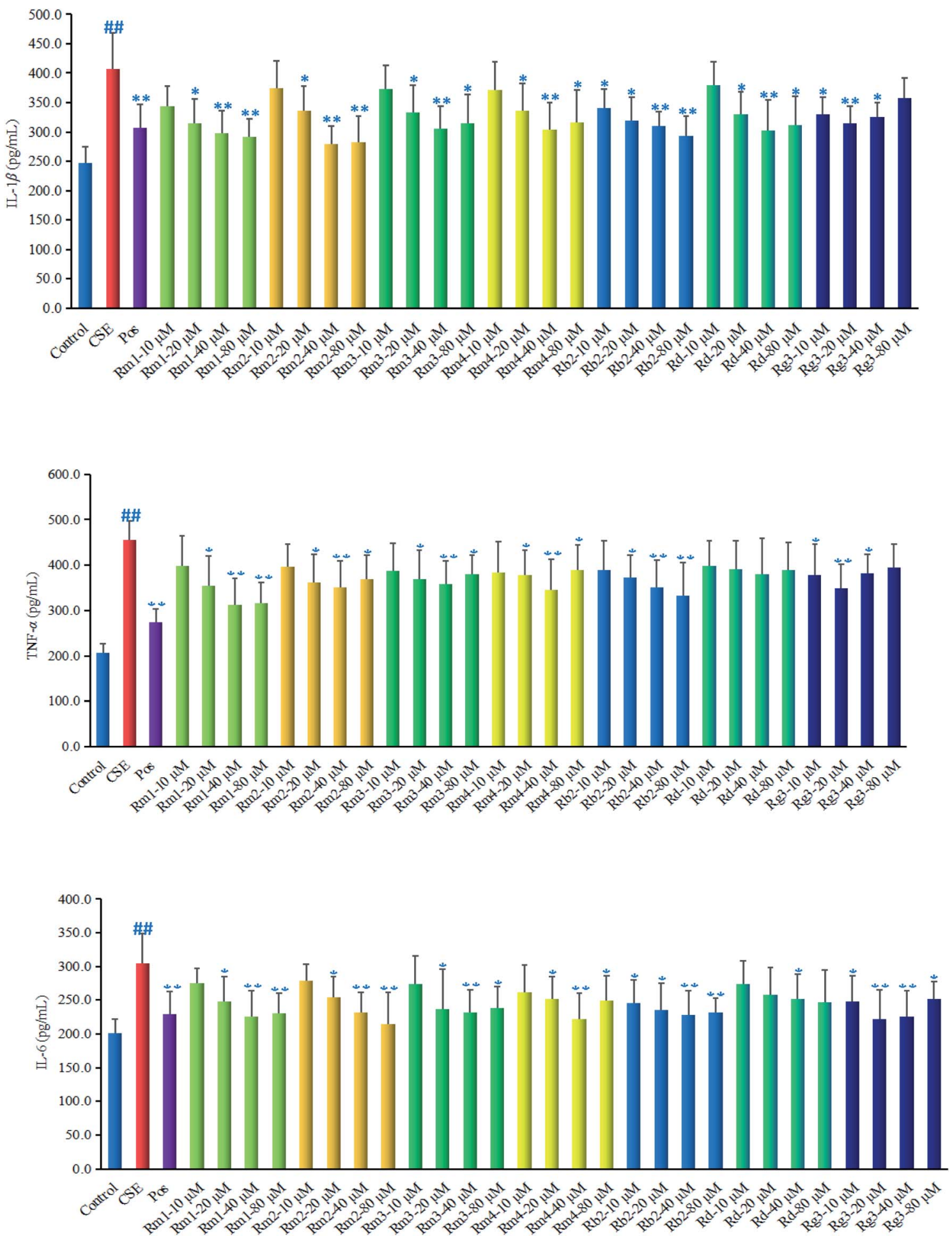

Fig. 4 Anti-inflammatory effect of compound 1-7 (10 $\mu \mathrm{M}, 20 \mu \mathrm{M}, 40 \mu \mathrm{M}$ and $80 \mu \mathrm{M})$ on the inflammatory cytokine tumor necrosis factor$\alpha$ (TNF- $\alpha$ ), interleukin-1 $\beta$ (IL-1 $\beta$ ) and interleukin-6 (IL-6) in CSE-exposed A549 cells. The results are expressed as mean \pm S.D., $n=6 .{ }^{\# \#} p<0.01$, compared with control group; ${ }^{* *} p<0.01$, compared with CSE group; $* p<0.05$, compared with CSE group.

hydroxyl group instead of being glycosylated. The location of hydroxyl group was determined by $\mathrm{HMBC}$ of 3 . In $\mathrm{HMBC}$ spectrum, the correlations between $\mathrm{H}-22$ with $\mathrm{C}-21$ and $\mathrm{C}-24, \mathrm{H}-$ 23 with C-20 and C-25, were observed. Thus, the structure of compound 3 was identified as 3-O-[ $\beta$-D-glucopyranosyl- $(1 \rightarrow 2)-\beta$ -
D-glucopyranosyl]-3 $\beta, 12 \beta, 20 S, 25$-tetrahydroxydammar-23-ene (ginsenoside Rm3, Fig. 1). Compound 3 was also a minor glucoside in wild ginseng. The complete signal assignments on basis of HMQC and HMBC were shown in Table 1. 

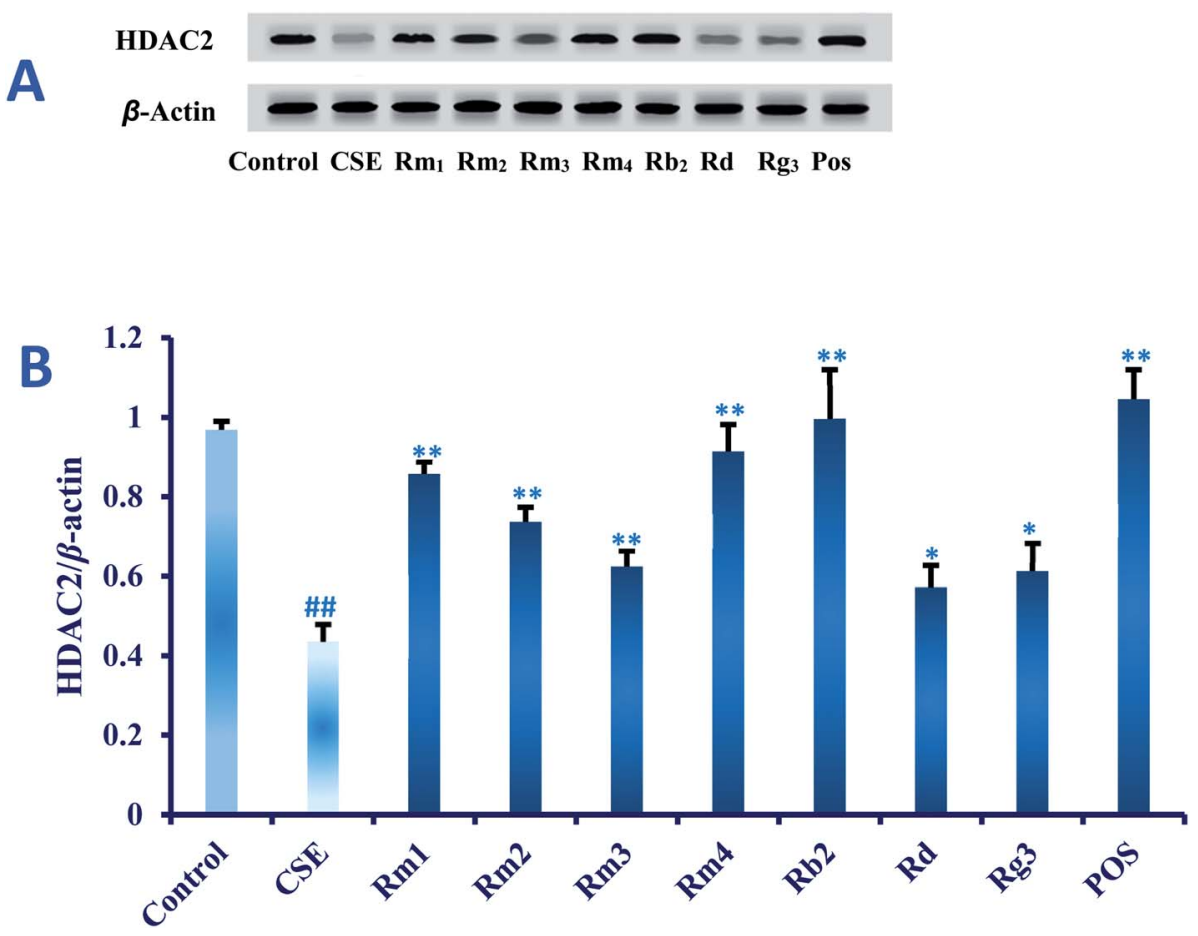

Fig. 5 (A) Effect of compound 1-7 on the protein expression of histone deacetylase 2 (HDAC2) in the CS-induced A549 cells examined by western blot analysis (B) quantitative analyses of HDAC2 in each group. Data are expressed as mean \pm SD $(n=6) .{ }^{\# \#} p<0.01$ as compared with control group, $* p<0.05, * * p<0.01$ as compared with CSE group.

Compound 4, a white amorphous powder $(\mathrm{MeOH})$, possessed a molecular formula of $\mathrm{C}_{45} \mathrm{H}_{74} \mathrm{O}_{16}$, as elucidated by the NMR spectra and HR-ESI-MS at $m / z$ 869.4890 $[\mathrm{M}-\mathrm{H}]^{-}$(calc. for 869.4899). The ${ }^{1} \mathrm{H}$ NMR spectrum of 4 displayed eight methyl signals at $\delta 0.82(3 \mathrm{H}, \mathrm{s}), 0.83(3 \mathrm{H}, \mathrm{s}), 0.83(3 \mathrm{H}, \mathrm{s}), 0.96$ $(3 \mathrm{H}, \mathrm{s}), 0.97$ (3H, s), $1.22(3 \mathrm{H}, \mathrm{s}), 1.34(3 \mathrm{H}, \mathrm{d}, J=6.5 \mathrm{~Hz}), 1.56$ $(3 \mathrm{H}, \mathrm{s})$ and $1.63(3 \mathrm{H}, \mathrm{d}, J=4.5 \mathrm{~Hz})$. The ${ }^{13} \mathrm{C}$ NMR spectrum of 4 revealed 45 signals, including ten methylene [one of them bearing an oxygen atom ( $\delta$ 61.49)], eighteen methine, eight quaternary carbons $[\delta 38.63,39.91,40.16,50.94,75.24,130.16$, 170.90 and 171.20], eight methyl carbons $(\delta 16.46,16.57,16.74$, 17.56, 17.80, 25.56, 26.38 and 31.00). The NMR data of 4 were similar to those of ginsenoside $\mathrm{Rg}_{2}{ }^{31}$ except a malonyl group was appeared in 4. The location of the malonyl group was determined by $\mathrm{HMBC}$ of 4 . The following correlations were found: $\mathrm{H}-21$ with $\mathrm{C}-17$ and $\mathrm{C}=\mathrm{O}, \mathrm{H}-17$ with $\mathrm{C}-21$ and $\mathrm{C}=\mathrm{O}$, which indicated that the malonyl group was linked to C-20. Thus, the structure of $\mathbf{4}$ was identified as 6-O-[ $\alpha$-L-rhamnopyranosyl-(1 $\rightarrow 2)$ - $\beta$-D-glucopyranosyl]-20-O-malonyl-dammar-24ene-3 $\beta, 6 \alpha, 12 \beta, 20 S$-tetraol (ginsenoside Rm4, Fig. 1). Compound 4 was also a minor glucoside in wild ginseng. The proton and carbon signals of $\mathbf{4}$ were fully assigned on the basis of HMQC and HMBC spectra (Table 1).

\subsection{Bioactivity evaluation}

Ginsenoside $\mathrm{Rg}_{1}$ (ref. 11) and $\mathrm{Rh}_{2}$ (ref. 12) had been reported to significantly inhibit COPD-related inflammation. As part of an ongoing effort to search for anti-COPD agents from wild ginseng, ${ }^{23}$ compounds 1-7 were evaluated for their anti- inflammatory effects on CSE-induced A549 cells. Dexamethasone was selected as positive drug (Pos). ${ }^{32}$

3.2.1 Cytotoxicity of cigarette smoke extract (CSE) and compounds 1-7 on the viability of A549 cells. The results of MTT showed that the cell viability of A549 cells was significantly affected $(p<0.01)$ by CSE $(\geqq 30 \%)$ (Fig. 3A). Therefore, in subsequent experiments, $20 \%$ CSE was chosen as stimulation. As shown in Fig. 3B, the cell viabilities of A549 cells were not significantly affected by $1-7$ at $10 \mu \mathrm{M}-80 \mu \mathrm{M}$. Then we evaluated the anti-inflammatory effects of $1-7$ at $10 \mu \mathrm{M}-80 \mu \mathrm{M}$ on CSEstimulated A549 cells.

3.2.2 Effect of compounds 1-7 on CSE-induced proinflammatory cytokine levels in A549 cells. The inflammatory development is characterized by the release of proinflammatory mediators such as tumor necrosis factor$\alpha$ (TNF- $\alpha$ ), interleukin-1 $\beta$ (IL-1 $\beta$ ) or interleukin-6 (IL-6), etc. Whether compounds 1-7 could inhibit the release of chemokine TNF- $\alpha$, IL-1 $\beta$ and IL-6 from CSE-induced lung epithelial cells was investigated in this paper. As shown in Fig. 4, TNF- $\alpha$, IL-1 $\beta$ and IL- 6 production markedly increase in the cell treated with CSE $(p<0.01)$, however, treated with compounds 1-7 could significantly reduce the levels of pro-inflammatory factors to various extents. Compound $\mathbf{1}(\mathrm{Rm} 1)$ could significantly reduce TNF- $\alpha$, IL-1 $\beta$ and IL-6 at $20(p<0.05), 40$ and $80(p<0.01) \mu \mathrm{M}$. Compound $2(\mathrm{Rm} 2)$ could significantly reduce IL-1 $\beta$ and IL-6 at $20(p<0.05), 40$ and $80(p<0.01) \mu \mathrm{M}$, significantly reduce TNF$\alpha$ at 20 and $80(p<0.05), 40(p<0.01) \mu \mathrm{M}$. Compound $3(\mathrm{Rm} 3)$ and Compound $4(\mathrm{Rm} 4)$ could significantly reduce IL-1 $\beta$, IL-6 and TNF- $\alpha$ at 20 and $80(p<0.05), 40(p<0.01) \mu \mathrm{M}$. 

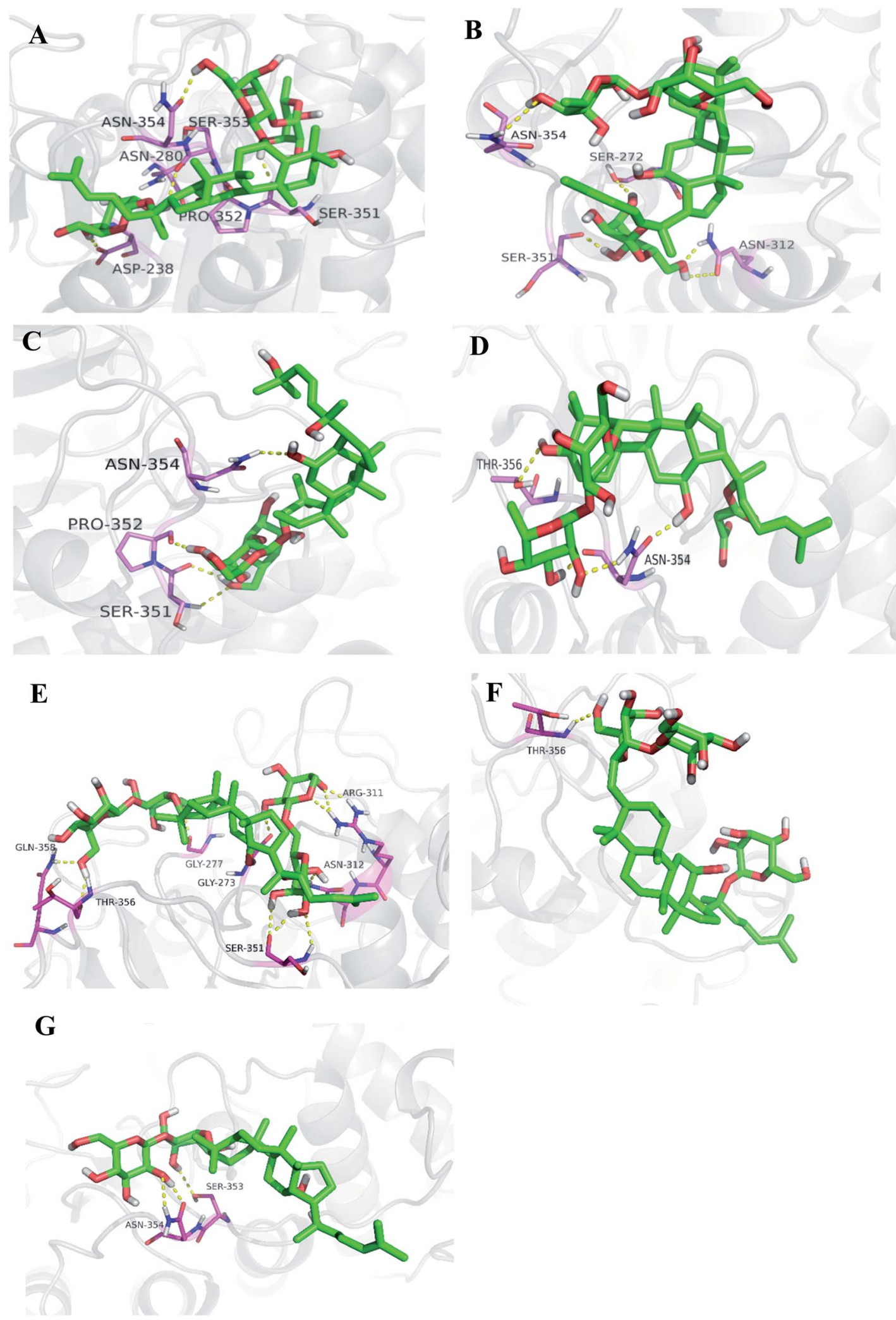

Fig. 6 Target of compound 1-7 against CSE-stimulated inflammatory response in A549 cells. (A-G) Interaction mode of compound 1-7 within binding pocket of HDAC2. (A-G) The hydrogen bonds represented as the yellow dashed lines.

Compound $5(\mathrm{Rb} 2)$ could significantly reduce IL-1 $\beta$ and IL-6 at 10 and $20(p<0.05), 40$ and $80(p<0.01) \mu \mathrm{M}$, reduce TNF- $\alpha$ at 20 $(p<0.05), 40$ and $80(p<0.01) \mu \mathrm{M}$. Compound $6(\mathrm{Rd})$ could significantly reduce IL-1 $\beta$ at 20 and $80(p<0.05), 40(p<0.01)$ $\mu \mathrm{M}$, reduce IL-6 at $80(p<0.05) \mu \mathrm{M}$, while had no significant effect on TNF- $\alpha$ level. Compound 7 (Rg3) could significantly 
reduce IL-1 $\beta$ and TNF- $\alpha$ at 10 and $40(p<0.05), 20(p<0.01) \mu \mathrm{M}$, reduce IL- 6 at 10 and $80(p<0.05), 20$ and $40(p<0.01) \mu \mathrm{M}$. The lowest effective concentration of compound 1, 2, 3, 4, 6 was all $20 \mu \mathrm{M}$, the lowest effective concentration of compound 5, 7 was both $10 \mu \mathrm{M}$. Meanwhile, the dose-dependent effect of the compounds was appeared with a certain range of 10-80 $\mu \mathrm{M}$. Interestingly, the activity of these compounds was not always increased with the increasing concentration, namely, when the concentration arrived a certain level, the activity might decrease to some extent.

3.2.3 Effect of compounds 1-7 on CSE-Mediated protein expression of HDAC2 in vitro. It has been demonstrated that histone deacetylase 2 (HDAC2) activity and egg self-expression in alveolar macrophages, peripheral lung tissues and bronchial tissues of COPD patients are significantly decreased. ${ }^{33,34}$ CSE can reduce HDAC2 activity and protein expression in rats, ${ }^{35}$ which can activate rat lung tissue NF- $k \mathrm{~B}$ pathway. ${ }^{36}$ Other studies have shown that CSE exposure can cause the decrease of HDAC2 protein and activity in A549 cell nucleus ${ }^{37}$ and activate the NF- $k \mathrm{~B}$ pathway in vivo. ${ }^{36}$

In order to explore the underlying mechanism of antiinflammatory property of compounds $1-7$, we investigated the effect of them on the protein expression of HDAC2 in CSEexposed A549 cells. In the present study, after 18 hours of CSE exposure in A549 cells, the expression of HDAC2 protein in each group of $40 \mu \mathrm{M}$ concentration was measured as shown in Fig. 5 . The results showed that the level of HDAC2 protein in A549 cells decreased significantly $(p<0.01)$ after CSE 18 hours exposure. Interestingly, treatment with each ginsenoside could significantly activated the expression of HDAC2 $(p<0.01, p<0.05)$ as compared to the CSE group (Fig. 5) in detail, which suggested that these compounds may up-regulated the expression of HDAC2 in CSE-exposed A549 cells.

3.2.4 Molecular docking study. The interactions between each ginsenoside and HDAC2 were visualized in molecular docking study. Ginsenoside Rm1 towards HDAC2 (PDB ID: 5IWG) gave six hydrogen bonds between Rm1 and six residues (ASN-354, ASN-280, SER-353, SER-351, PRO-352 and ASP-238) (Fig. 6A), Rm2 towards HDAC2 (PDB ID: 5IWG) displayed five hydrogen bonds between Rm2 and four residues (ASN-354, SER-351, ASN-312 and SER-272) (Fig. 6B), Rm3 towards HDAC2 (PDB ID: 5IWG) showed four hydrogen bonds between Rm3 and three residues (ASN-354, SER-351 and PRO-352) (Fig. 6C), Rm4 towards HDAC2 (PDB ID: 5IWG) exhibited four hydrogen bonds between Rm4 and two residues (THR-356 and ASN-354) (Fig. 6D). $\mathrm{Rb}_{2}$ towards HDAC2 (PDB ID: 5IWG) exhibited seven hydrogen bonds between $\mathrm{Rb}_{2}$ and seven residues (ARG-311, ASN-312, SER-351, GLY-273, GLY-277, THR-356, GLN-358) (Fig. 6E), Rd towards HDAC2 (PDB ID: 5IWG) exhibited one hydrogen bonds between Rd and one residues (THR-356) (Fig. 6F), $\mathrm{Rg}_{3}$ towards HDAC2 (PDB ID: 5IWG) exhibited two hydrogen bonds between $\mathrm{Rg}_{3}$ and two residues (SER-353 and ASN-354) (Fig. 6G). These results were consistent with the anti-inflammatory effects of the seven ginsenosides in CSE-induced inflammatory response in A549 cells. These docking results illustrated that the ginsenosides may directly bind to HDAC2 to exert lung protective effects due to strong hydrogen bonding effects.

\section{Conclusions}

In the present study, four minor previously undescribed ginsenosides, named as ginsenoside Rm1 (1), Rm2 (2), Rm3 (3) and $\mathrm{Rm} 4$ (4), along with five known ginsenosides, $\mathrm{Rb}_{2}(5), \mathrm{Rd}(6), \mathrm{Rg}_{3}$ (7), $\operatorname{Rg}_{1}$ (8) and $\mathrm{Rh}_{2}$ (9) were isolated from wild ginseng by a UPLC-QTOF-MS-guided fractionation procedure. Their structures were elucidated on the basis of spectroscopic and spectrometric data (1D and 2D NMR, IR, and HR-ESI-MS). The antiCOPD activities were investigated using CSE-stimulated A549 cells. Compounds 1-7 could ameliorate inflammatory reaction in vitro. Furthermore, the protective effect against CSE could be related to the HDAC2 pathway. The study provided some evidences to further elucidate the chemical composition of wild ginseng, and revealed that the compounds isolated from wild ginseng had the protective effect on the injury of cigarette smoke in vitro. The study provides a theoretical basis for the further utilization and development of the wild ginseng. It reminds us that it is meaningful to explore the protective effect of the wild ginseng against the COPD in vivo.

\section{Conflicts of interest}

The authors declare no conflicts of interest.

\section{Acknowledgements}

This work was financially supported by the Bethune Plan Research Project of Jilin University (Grant 2018B22) and the Graduate Innovation Fund of Jilin University (Grant 101832018C085).

\section{Notes and references}

1 D. Xiao, H. Yue, Y. Xiu, et al., Accumulation characteristics and correlation analysis of five ginsenosides with different cultivation ages from different regions, J. Ginseng Res., 2015, 39, 338.

2 M. Mizuno, J. Yamada, H. Terai, et al., Differences in immunomodulating effects between wild and cultured Panax ginseng, Biochem. Biophys. Res. Commun, 1994, 200, 1672.

3 H. L. Zhu, H. Q. Lin, J. Tan, C. Z. Wang, et al., UPLC-QTOF/ MS-based nontargeted metabolomic analysis of mountainand garden-cultivated Ginseng of different ages in Northeast China, Molecules, 2019, 24, 33.

4 The Global Initiative for Chronic Obstructive Lung Disease (GOLD), Global Strategy for the Diagnosis Management and Prevention of COPD2019, available from: http:/goldcopd.org.

5 P. R. Sandra, L. D. Puerto-Nevado, T. E. Raúl, et al., Role of Recently Migrated Monocytes in Cigarette Smoke-Induced Lung Inflammation in Different Strain of Mice, PLoS One, 2013, 8, e72975.

$6 \mathrm{~J}$. L. Wright, M. Cosio and A. Churg, Animal Models of Chronic Obstructive Pulmonary Disease, Drug Res., 2011, 51, 1004. 
7 N. Kim, K. Kim, B. Y. Choi, et al., Metabolomic Approach for Age Discrimination of Panax ginseng Using UPLC-Q-Tof MS, J. Agric. Food Chem., 2011, 59, 10435.

8 N. Kim, K. Kim, D. H. Lee, et al., Nontargeted metabolomics approach for age differentiation and structure interpretation of age-dependent key constituents in hairy roots of Panax ginseng, J. Nat. Prod., 2012, 75, 1777.

9 L. Wu, A. L. Zhang, Y. M. Di, et al., Panax ginseng therapy for chronic obstructive pulmonary disease: a clinical trial protocol and pilot study, Chin. Med., 2014, 9, 20.

10 D. Gross, Z. Shenkman, B. Bleiberg, et al., Ginseng improves pulmonary functions and exercise capacity in patients with COPD, Monaldi Arch. Chest Dis., 2002, 57, 242.

11 S. Guan, Q. Liu, F. Han, et al., Ginsenoside Rg1 ameliorates cigarette smoke-induced airway fibrosis by suppressing the TGF- $\beta 1 /$ Smad pathway in vivo and in vitro, Biomed. Res., 2017, 2, 1 .

12 W. Y. Bi, B. D. Fu, H. Q. Shen, et al., Sulfated Derivative of 20(S)-Ginsenoside Rh2 Inhibits Inflammatory Cytokines Through MAPKs and NF-kappa B Pathways in LPS-Induced RAW264.7, Macrophages, Inflammation, 2012, 35, 1659.

13 S. H. Jung, M. S. Woo, S. Y. Kim, et al., Ginseng saponin metabolite suppresses phorbol ester-induced matrix metalloproteinase-9 expression through inhibition of activator protein-1 and mitogen-activated protein kinase signaling pathways in human astroglioma cells, Int. J. Cancer, 2010, 118, 490.

14 D. Malhotra, R. K. Thimmulappa, N. Mercado, et al., Denitrosylation of HDAC2 by targeting Nrf2 restores glucocorticosteroid sensitivity in macrophages from COPD patients, J. Clin. Invest., 2011, 121, 4289.

15 P. J. Barnes, Corticosteroid resistance in patients with asthma and chronic obstructive pulmonary disease, $J$. Allergy Clin. Immunol., 2013, 131, 636.

16 A. Hakim, I. M. Adcock and O. S. Usmani, Corticosteroid resistance and novel anti-inflammatory therapies in chronic obstructive pulmonary disease, Drugs, 2012, 72, 1299.

17 J. P. Barnes, Development of new drugs for COPD, Curr. Med. Chem., 2013, 20, 1531.

18 M. Yokode, T. Kita, H. Arai, et al., Cholesteryl ester accumulation in macrophages incubated with low density lipoprotein pretreated with cigarette smoke extract, Proc. Natl. Acad. Sci. U. S. A., 1988, 85, 2344.

19 Y. Yamaguchi, S. Kagota, J. Haginak, M. Kunitomo, et al., Participation of peroxynitrite in oxidative modification of LDL by aqueous extracts of cigarette smoke, FEBS Lett., 2002, 512, 218.

20 L. Chen, Q. Ge, G. T. Jin, H. Alkhouri, et al., Effects of cigarette smoke extract on human airway smooth muscle cells in COPD, Eur. Respir. J., 2014, 44, 634.

21 M. Bak, J. S. G. Hong, J. W. Lee, et al., Red ginseng marc oil

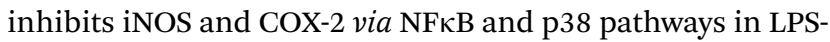
stimulated RAW 264.7 macrophages, Molecules, 2012, 17, 13769.
22 Z. Qi, W. Li, J. Tan, et al., Effect of ginsenoside Rh2 on renal apoptosis in cisplatin-induced nephrotoxicity in vivo, Phytomedicine, 2019, 61, 152862.

23 Z. Qi, Z. Li, X. W. Guan, et al., Four Novel Dammarane-Type Triterpenoids from Pearl Knots of Panax ginseng Meyer cv. Silvatica, Molecules, 2019, 24, 1159.

24 J. G. Cho, M. K. Lee, J. W. Lee, et al., Physicochemical Characterization and NMR Assignments of Ginsenosides $\mathrm{Rb} 1, \mathrm{Rb} 2, \mathrm{Rc}$, and Rd Isolated from Panax ginseng, $J$. Ginseng Res., 2010, 34, 113.

25 M. C. Yang, D. S. Seo, J. K. Hong, et al., Ginsenosides from the roots of Korean cultivated-wild ginseng, Nat. Prod. Sci., 2008, 14, 171.

26 B. D. Fu, W. Y. Bi, C. L. He, et al., Sulfated derivatives of 20 $(S)$-ginsenoside Rh2 and their inhibitory effects on LPSinduced inflammatory cytokines and mediators, Fitoterapia, 2013, 84, 303.

27 H. X. Sun, Y. Chen and Y. Ye, Ginsenoside Re and Notoginsenoside $\mathrm{R}_{1}$ : Immunologic Adjuvants with Low Haemolytic Effect, Chem. Biol., 2006, 3, 718.

28 L. Tu, Y. Zhao, Z. Y. Yu, et al., Six New Dammarane Triterpenoids from Viburnum cylindricum, Helv. Chim. Acta, 2010, 91, 1578.

29 C. Z. Gu, J. J. Lv, X. X. Zhang, et al., Minor dehydrogenated and cleavaged dammarane-type saponins from the steamed roots of Panax notoginseng, Fitoterapia, 2015, 103, 97.

30 M. Yoshikawa, T. Murakami, T. Ueno, et al., Bioactive saponins and glycosides. VIII. Notoginseng (1): new dammarane-type triterpene oligoglycosides, notoginsenosides-A, -B,-C, and-D, from the dried root of Panax notoginseng (Burk.) FH, Chen, Chem. Pharm. Bull, 1997, 45, 1039.

31 R. Teng, H. Li, J. Chen, et al., Complete assignment of ${ }^{1} \mathrm{H}$ and ${ }^{13} \mathrm{C}$ NMR data for nine protopanaxatriol glycosides, Magn. Reson. Chem., 2002, 40, 483.

32 C. H. Ma, L. He, Z. Wang, et al., Betulin inhibited cigarette smoke-induced COPD in mice, Biomed. Pharmacother., 2017, 85, 679.

33 P. J. Barnes and I. M. Adcock, Glucocorticoid resistance in inflammatory diseases, Lancet, 2009, 373, 1905.

34 P. J. Barnes, Role of HDAC2 in the Pathophysiology of COPD, Annu. Rev. Physiol., 2009, 71, 451.

35 J. A. Marwick, P. A. Kirkham, C. S. Stevenson, et al., Cigarette smoke alters chromatin remodeling and induces proinflammatory genes in rat lungs, Am. J. Respir. Cell Mol. Biol., 2004, 31, 633.

36 L. Zeng, J. C. Dong, W. Q. Yu, et al., Baicalin attenuates inflammation by inhibiting $\mathrm{NF}-\kappa \mathrm{B}$ activation in cigarette smoke induced inflammatory models, Pulm. Pharmacol. Ther., 2010, 23, 411.

37 F. M. Moodie, J. A. Marwick, C. S. Anderson, et al., Oxidative stress and cigarette smoke alter chromatin remodeling but differentially regulate NF-kB activation and proinflammatory cytokine release in alveolar epithelial cells, FASEB J., 2004, 18, 1897. 